\title{
Improving Bandwidth of Yagi-Uda Arrays
}

\section{Richard A. Formato}

Cataldo \& Fisher, LLC, Woburn, USA.

Email: rf2@ieee.org

Received October $13^{\text {th }}, 2011$; revised October $30^{\text {th }}, 2011$; accepted November $18^{\text {th }}, 2011$

\begin{abstract}
A novel approach for improving antenna bandwidth is described using a 6-element Yagi-Uda array as an example. The new approach applies Central Force Optimization, a deterministic metaheuristic, and Variable $Z_{0}$ technology, a novel, proprietary design and optimization methodology, to produce an array with $33.09 \%$ fractional impedance bandwidth. This array's performance is compared to its CFO-optimized Fixed $Z_{0}$ counterpart, and to the performance of a 6-element Dominating Cone Line Search-optimized array. Both CFO-optimized antennas exhibit better performance than the DCLS array, especially with respect to impedance bandwidth. Although the Yagi-Uda antenna was chosen to illustrate this new approach to antenna design and optimization, the methodology is entirely general and can be applied to any antenna against any set of performance objectives.
\end{abstract}

Keywords: Variable $Z_{0} ; Z_{0}$; Characteristic Impedance; Feed System; Antenna; Antenna Design; Antenna Optimization; Design Objectives; Performance Objectives; Bandwidth; Impedance Bandwidth; Broadband; Ultra Wideband; UWB; Yagi; Yagi-Uda; Central Force Optimization; CFO; Numerical Optimization; Optimization Algorithm; Metaheuristic; Dominating Cone Line Search; DCLS

\section{Introduction}

This note describes a novel approach to improving antenna bandwidth using a six-element Yagi-Uda array as an example. Developed more than 85 years ago [1,2], the "Yagi" still is widely used, but it is inherently narrowband, to quote: "Usually Yagi-Uda arrays have low input impedance and relatively narrow bandwidth (on the order of about 2\%)" [3, p.396]. Modern well-designed Yagis achieve greater bandwidth, on the order of 5\% [4] to more than $15 \%$ [5], but these bandwidths still are far below the requirements of many wireless systems. The Federal Communications Commission, for example, defines an Ultra Wideband (UWB) antenna as having a fractional impedance bandwidth (IBW) of at least 20\%, or an absolute bandwidth of $500 \mathrm{MHz}$ [6, p.15]. Central Force Optimization (CFO) and Variable $Z_{0}^{(\mathrm{sm})}{ }^{1}$ technology (Var $\left.Z_{0}, V Z_{0}\right)$ are applied to the Yagi design problem, and the array's IBW performance is compared to another state-of-the-art design. The CFO- $V Z_{0}$ Yagi achieves a very robust IBW of $33.09 \%$ for VSWR $\leq 2: 1$.

\section{Yagi-Uda Array}

The 6-element array comprises a center-fed driven ele-

${ }^{1}$ Variable $Z_{0}, \mathrm{~V} Z_{0}$, and $\operatorname{Var} Z_{0}$ are trademarks and service marks of Massachusetts Corporation Variable $Z_{0}$, Ltd., P.O. Box 1714, Harwich, MA 02645 USA. ment (DE) excited by a radio-frequency (RF) source flanked by a parasitic reflector (REF) on one side and four parasitic directors $\left(D_{n}, n=1, \cdots, 4\right)$ on the other. All elements are PEC (Perfectly Electrically Conducting). Three Yagis are described in this paper. Two were optimized with CFO, one using Variable $Z_{0}$, which treats $Z_{0}$ as an unknown variable whose value is to be determined by the optimization algorithm $[7,8]$, and the other using the traditional approach of assigning $Z_{0}$ a fixed value (50 $\Omega$ resistive in this case). These two antennas are referred to, respectively, as $\mathrm{CFO}-V Z_{0}$ and $\mathrm{CFO}-F Z_{0}$. $\mathrm{CFO}$ was selected as the optimization algorithm because it has performed well against recognized benchmark functions and problems in applied electromagnetics [9-18] and is a useful tool for antenna optimization.

The third antenna was optimized with Dominating Cone Line Search (DCLS) [5] and therefore provides a state-of-the-art comparison. The "A3" Yagi, whose dimensions are adapted from in table IV in [5] (REF moved to the Y-axis), was chosen for comparison because it has the largest bandwidth of the three antennas discussed in that paper. Both CFO and DCLS are deterministic algorithms, thus providing the major advantage compared to stochastic algorithms of returning the same results for every run with the same setup.

The Yagis' performance was computed using NEC4.2D (Numerical Electromagnetics Code ver. 4.2 double 
precision) [19-21]. NEC is a widely used Method of Moments (MoM) wire structure modeling program developed at Lawrence Livermore National Laboratory (LLNL). A freeware version of NEC-2 is available online (source code, executables and a GUI) [22,23]. The modeled antennas are shown in Figure 1 (visualized using 4nec2 [22], the red circle indicating the RF source with the axis length being 1 meter for scaling). Each design's geometry is quite different.

Table 1 shows element lengths and positions along the boom ( $+\mathrm{X}$-axis). Dimensions are in wavelengths, $\lambda_{0}$, at the design frequency, $f_{0}$. The corresponding NEC input files appear in Figure 2. Because NEC requires dimen-

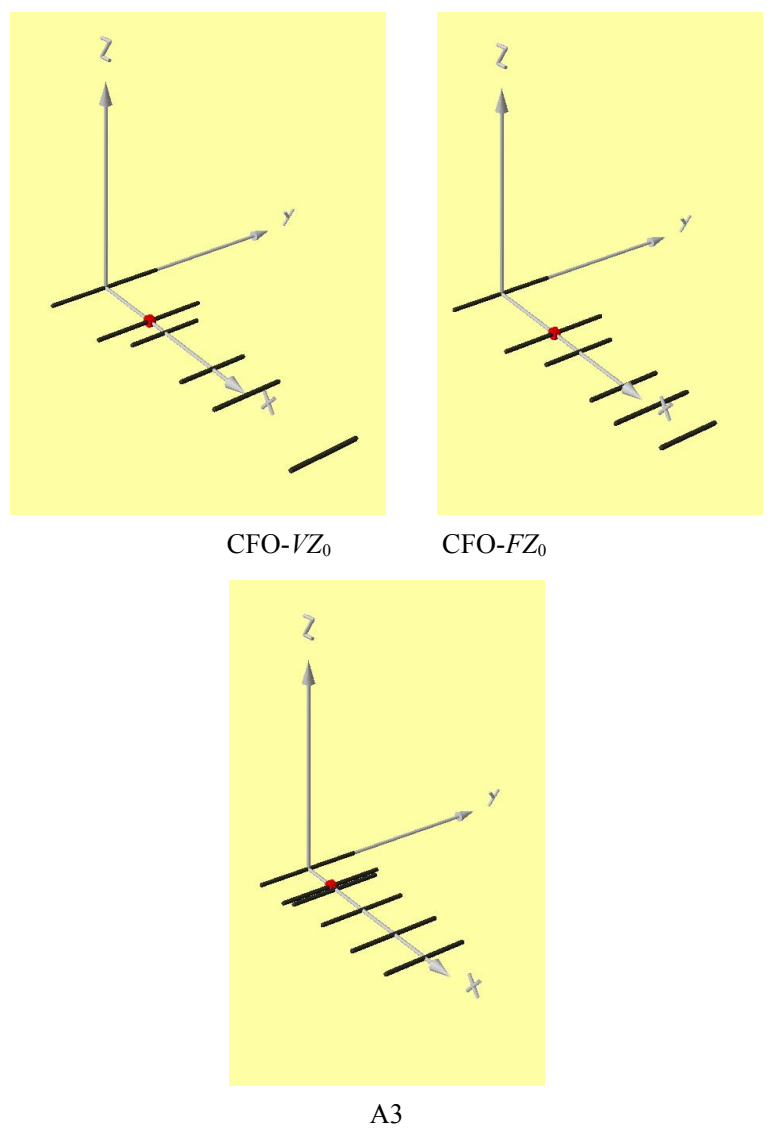

Figure 1. 6-element yagis.

Table 1. Yagi element lengths \& boom positions.

\begin{tabular}{ccccccc}
\hline \multirow{2}{*}{ Array Element } & \multicolumn{2}{c}{$\mathrm{CFO}-V Z_{0}$} & \multicolumn{2}{c}{$\mathrm{CFO}-F Z_{0}$} & \multicolumn{2}{c}{$\mathrm{A} 3$} \\
\cline { 2 - 7 } & $L\left(\lambda_{0}\right)$ & $X\left(\lambda_{0}\right)$ & $L\left(\lambda_{0}\right)$ & $X\left(\lambda_{0}\right)$ & $L\left(\lambda_{0}\right)$ & $X\left(\lambda_{0}\right)$ \\
\hline $\mathrm{REF}$ & 0.578 & 0 & 0.516 & 0 & 0.5072 & 0 \\
$\mathrm{DE}$ & 0.540 & 0.348 & 0.508 & 0.410 & 0.5070 & 0.1808 \\
$D_{1}$ & 0.346 & 0.461 & 0.340 & 0.585 & 0.4416 & 0.2144 \\
$D_{2}$ & 0.324 & 0.789 & 0.334 & 0.885 & 0.4169 & 0.4159 \\
$D_{3}$ & 0.332 & 1.006 & 0.364 & 1.056 & 0.4325 & 0.6397 \\
$D_{4}$ & 0.326 & 1.443 & 0.270 & 1.267 & 0.3952 & 0.8405 \\
\hline
\end{tabular}

sions in meters, $f_{0}$ was chosen to be $299.8 \mathrm{MHz}$ corresponding to a wavelength of $\lambda_{0}=1$ meter. The Yagi designs therefore can be scaled to any frequency because dimensions in meters are in wavelengths at $f_{0}$. The element radius was set to 0.009097 meter (or $\lambda_{0}$ ) because that value is used in [5]. Note that the design frequency $f_{0}$ and the band center frequency, $f_{C}$, discussed below are not (necessarily) the same. Even though the array is designed at a particular frequency, the optimized design's best performance may be (likely is) in a band whose center frequency is different.

\section{CFO-Variable $Z_{0}$}

The objective function (fitness) to be maximized by CFO was defined as

$$
F(\vec{x})=\sum_{k=1}^{3} c_{k} \cdot G_{f w d}\left(f_{k}, \vec{x}\right)-\sum_{i=1}^{3} c_{i} \cdot \operatorname{VSWR} / / Z_{0}\left(f_{i}, \vec{x}\right)
$$

where $f$ is the frequency and $\vec{x}$ the decision vector defined as

$$
\begin{gathered}
\vec{x}=\left(Z_{0}, L_{R E F}, L_{D E}, L_{D_{1}}, L_{D_{2}}, L_{D_{3}}, L_{D_{4}},\right. \\
\left.X_{D E}, X_{D_{1}}, X_{D_{2}}, X_{D_{3}}, X_{D_{4}}\right)
\end{gathered}
$$

The Yagi optimization problem is 12-dimensional with the following decision variables: $Z_{0}$, the feed system characteristic impedance (or source internal impedance if there is no feed line); and the eleven geometric variables corresponding to the Yagi element lengths (" $L ")$ and boom coordinates (" $X$ "), each subscripted with the corresponding element name (note that REF is placed symmetrically on the $Y$-axis, that is, at $X=0$ ). The fitness increases with increasing forward gain $G_{f w d}$ and decreasing VSWR $/ / Z_{0}$, VSWR $/ / Z_{0}$ being the voltage standing wave ratio relative to $Z_{0}$ (// denotes "relative to"). The fitness is evaluated at three frequencies as shown in Table 2 using the empirically determined coefficients $c_{i}$ and $c_{k} . G_{f w d}$ is evaluated in the direction of the $+\mathrm{X}$-axis $\left(\theta=90^{\circ}, \varphi=0^{\circ}\right.$ in NEC's right-handed spherical polar coordinate system). Algorithm details and a complete source code listing are available online [8], and an electronic listing is available upon request to the author (rf2@ieee.org).

The objective function is designed to maximize gain and bandwidth. The term "bandwidth" refers generally to the range of frequencies over which some specific an-

Table 2. Fitness coefficients.

\begin{tabular}{ccccc}
\hline$i$ & $c_{i}$ & $k$ & $c_{k}$ & $f_{i, k}(M H z)$ \\
\hline 1 & 5.0 & 1 & 0.2 & 239.8 \\
2 & 8.0 & 2 & 0.8 & 299.8 \\
3 & 0.9 & 3 & 1.0 & 359.8 \\
\hline
\end{tabular}



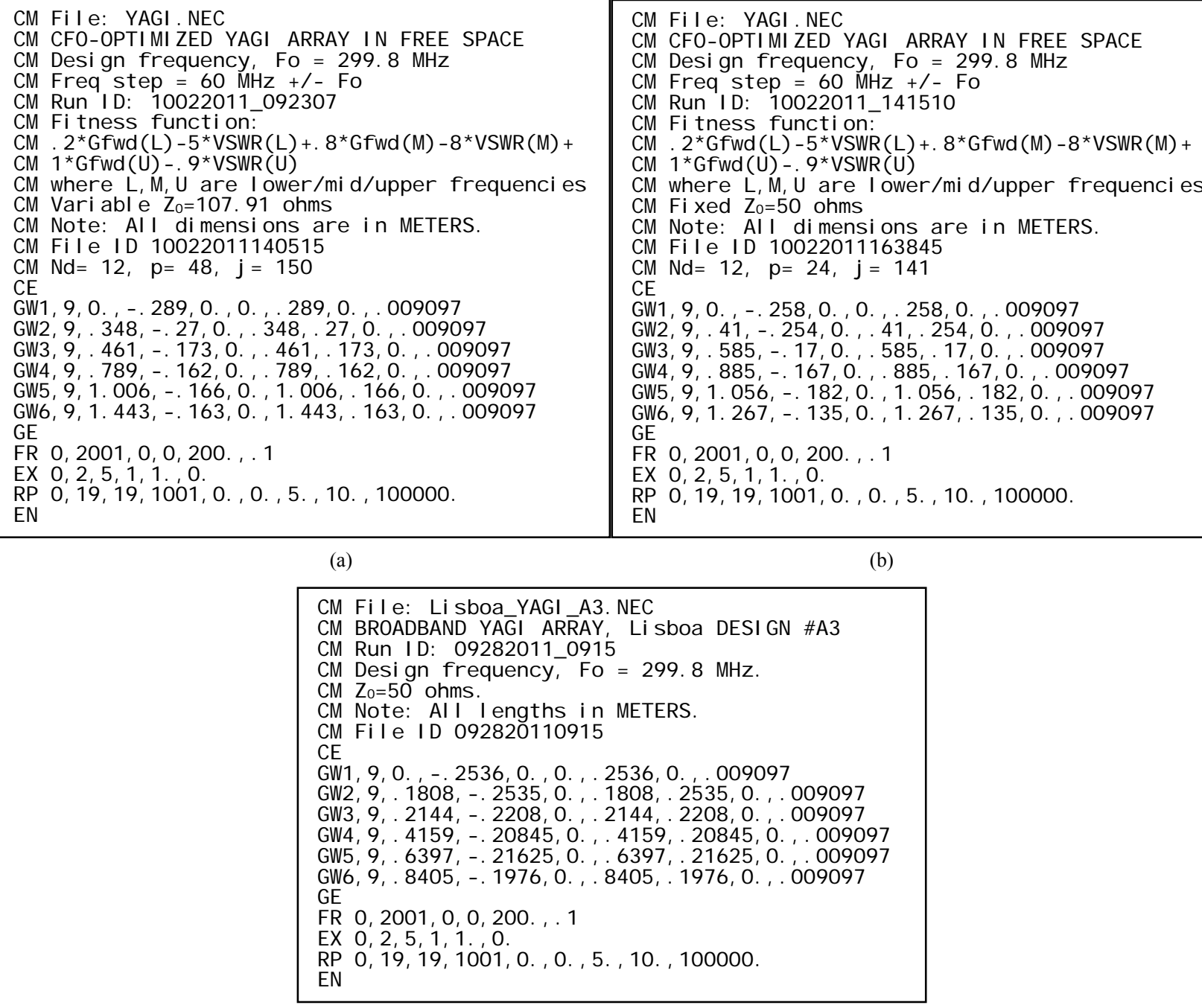

(c)

Figure 2. (a) $\mathrm{CFO}-\mathrm{VZ}_{0}$; (b) $\mathrm{CFO}-\mathrm{FZ}_{0}$; (c) A3.

tenna performance measure is met. For example, "gain bandwidth" is the frequency range over which a minimum power gain is achieved, and so on with respect to other performance measures. IBW is defined as the frequency band or bands within which the antenna input impedance, $Z_{i n}=R_{i n}+j X_{i n}, j=\sqrt{-1}$, is matched to the feed system characteristic impedance, $Z_{0}$, within specified limits. The desired degree of matching can be specified in many ways, for example, in terms of the antenna's actual input impedance (resistance, $R_{i n}$, and reactance, $X_{i n}$ ) as a function of frequency, or, as is more often the case in practice, in terms of a maximum VSWR. IBW typically is specified as VSWR // $Z_{0} \leq 2: 1$, which is equivalent to a return loss (scattering parameter $S_{11}$ ) approximately less than $-10 \mathrm{~dB}$. Other VSWR thresholds can be used instead, and frequently are. Military systems, for example, often use a 3:1 threshold. Zehforoosh et al. [24] describe the design of an UWB microstrip antenna and provide a good summary of $Z_{0}$ 's significance as a design parameter in the context of IBW.

Of course, the antenna designer is free to specify any desired fitness function, and its specific form will produce different antenna designs as a consequence of the different decision space landscape. Some objective functions may introduce $Z_{0}$ indirectly as a variable quantity, as is done here through the VSWR; or it may be introduced explicitly as, for example, in the bowtie fitness function in [7], where it is variable, or in the second and third objective functions used in [25], where it is fixed.

The objective of maximizing IBW is more easily met using Variable $Z_{0}$ instead of traditional methodology. Var $Z_{0}$ is a new, proprietary (patent pending) paradigm for antenna design and optimization methodology that apparently has been heretofore overlooked. For purposes of discussion the term "design" refers to the process of specifying a complete set of parameters defining an an- 
tenna meeting specific performance objectives, while "optimization" refers to specifying a complete set of parameters defining the antenna that best meets specific performance objectives. Methodology refers to the methods, techniques, processes, or procedures traditionally used for antenna design and optimization that treat $Z_{0}$ as a fixed parameter with a constant value that is assigned at the start of the methodology (even if multiple parametric runs are made). Traditional methodology does not consider $Z_{0}$ a variable quantity whose value is determined by the methodology. This distinction is fundamental and quite important. Traditional methodology excludes from the outset all designs that could provide better performance by using some other value of $Z_{0}$. The A3 array, for example, was designed against $Z_{0}=50 \Omega$ resistive, but its performance likely would be better with another value. By treating $Z_{0}$ as another design variable in the set of variable antenna system parameters to be determined by the methodology, Var $Z_{0}$ improves on traditional methodology by adding another degree of freedom to the design space or, in the case of optimization, the decision space, thereby making it easier to achieve any particular performance objectives. While $\operatorname{Var} Z_{0}$ is especially useful for increasing IBW, it can be used to achieve any performance objectives, including ones not involving IBW.

Besides CFO, any number of other commonly employed algorithms, such as Particle Swarm (PSO) [26], Ant Colony (ACO) [27], Group Search Optimizer (GSO) [28], Differential Evolution (DE) [29-31], or Genetic Algorithm (GA) [32] could be used instead. This "product by process" approach applies to any methodology, deterministic ones like $\mathrm{CFO}$; stochastic metaheuristics like PSO, ACO, GSO, DE or GA; analytic approaches such as extended Wu-King impedance loading [14]; or even "seat of the pants" design or optimization based on experience, intuition, or a "best guess." The specific design or optimization methodology is irrelevant to the novelty and utility of treating $Z_{0}$ as a design variable instead of a fixed parameter. Var $Z_{0}$ can be used advantageously with any design or optimization methodology.

\section{Results}

Figure 3 plots VSWR for the three Yagis, and Table $\mathbf{3}$ summarizes the bandwidth data for three different VSWR thresholds $(2: 1,2.5: 1$, and $3: 1)$. In the table, $f_{L}$ and $f_{U}$ are the lower and upper frequency limits (MHz) corresponding to VSWRs not exceeding the specified threshold, and $\Delta f=f_{U}-f_{L}$ is the bandwidth in MHz. The fractional bandwidth in percent relative to the band center frequency $f_{C}$ is computed as

$\mathrm{BW}(\%)=\frac{200 \Delta f}{f_{L}+f_{U}}$. The CFO-Var $Z_{0}$ Yagi exhibits

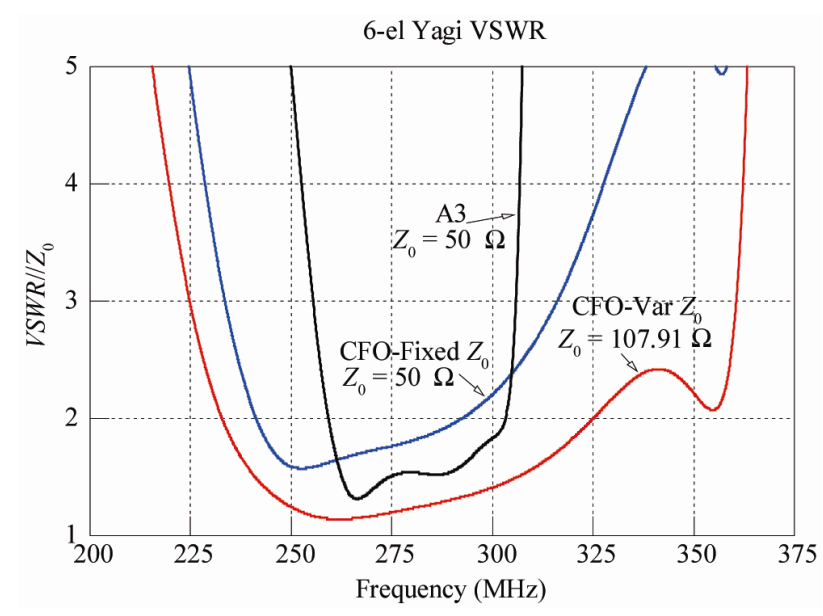

Figure 3. Yagi VSWR relative to $Z_{0}$.

Table 3. 6-el Yagi bandwidth.

\begin{tabular}{cccccc}
\hline \multicolumn{7}{c}{ VSWR Threshold 2:1 } \\
\hline Yagi Design & $f_{L}$ & $f_{C}$ & $f_{U}$ & $\Delta f$ & BW (\%) \\
${\text { CFO- } V Z_{0}^{(1)}}^{(1)}$ & 232.80 & 278.95 & 325.10 & 92.30 & 33.09 \\
${\text { CFO- } F Z_{0}^{(2)}}^{(2)}$ & 241.30 & 266.95 & 292.60 & 51.30 & 19.22 \\
A3 $^{(3)}$ & 259.25 & 281.23 & 303.20 & 43.95 & 15.63 \\
\hline \multicolumn{7}{c}{ VSWR Threshold 2.5:1 } \\
\hline Yagi Design & $f_{L}$ & $f_{C}$ & $f_{U}$ & $\Delta f$ & BW (\%) \\
CFO- $V Z_{0}$ & 228.15 & 293.63 & 359.10 & 130.95 & 44.60 \\
CFO- $F Z_{0}$ & 236.80 & 272.15 & 307.50 & 70.70 & 25.98 \\
A3 & 257.10 & 281.08 & 305.05 & 47.95 & 17.06 \\
\hline \multicolumn{7}{c}{ VSWR Threshold 3:1 } \\
Yagi Design & $f_{L}$ & $f_{C}$ & $f_{U}$ & $\Delta f$ & BW (\%) \\
CFO- $V Z_{0}$ & 224.80 & 292.70 & 360.60 & 135.80 & 46.40 \\
CFO- $F Z_{0}$ & 233.55 & 274.83 & 316.10 & 82.55 & 30.04 \\
A3 & 255.40 & 280.62 & 305.83 & 50.43 & 17.97 \\
\hline
\end{tabular}

${ }^{(1)} \mathrm{CFO}$-optimized array, Variable $Z_{0}, Z_{0}=107.91 \Omega .{ }^{(2)} \mathrm{CFO}$-optimized array, Fixed $Z_{0}, Z_{0}=50 \Omega .{ }^{(3)}$ DCLS-optimized array (Lisboa A3), Fixed $Z_{0}, Z_{0}=$ $50 \Omega$

UWB performance (fractional bandwidth $\geq 20 \%$ ) at all VSWR thresholds, whereas the fixed $Z_{0}$ antenna is UWB at 2.5:1 and above. The Var $Z_{0}$ approach increased the Yagi's $V S W R \leq 2: 1$ IBW from $19.22 \%$ at $Z_{0}$ $=50 \Omega$ to $33.09 \%$ at $Z_{0}=107.91 \Omega$. This improvement is dramatic, and directly attributable to Var $Z_{0}$ because CFO is a deterministic metaheuristic (the improvement thus cannot be a consequence of an optimizer's stochasticity). With respect to matching this array to a "standard" $50 \Omega$ feed system impedance, a 2.16:1 impedance ratio broadband transformer or other suitable matching network is required, which easily is accomplished with state-of-the-art matching techniques. By injecting an additional degree of freedom into the traditional antenna design or optimization methodology, $\operatorname{Var} Z_{0}$ technol- 
ogy has produced a substantially better antenna than the CFO-optimized traditional fixed $Z_{0}$ design.

Compared to the CFO-optimized Yagis, the $\mathrm{A} 3$ array is considerably more narrowband. Its VSWR $\leq 2: 1 \mathrm{IBW}$ is only $15.63 \%$, and it increases only slightly to $17.97 \%$ at 3:1. The A3 array's best IBW performance across all three VSWR thresholds is not as good as the CFO-FZ array at its lowest threshold. This is a consequence of the very steep skirts in its VSWR plot. While the CFO-Var $Z_{0}$ array exhibits similarly steep skirts, as is evident from the plot, its basic IBW is much greater to start.

Figures $\mathbf{4}$ and $\mathbf{5}$ plot forward gain $(\mathrm{dBi}$, decibels relative to isotropic) and front-to-back ratio (FBR), respectively [note that in this case directivity power gain are equal because the PEC array elements result in 100\% radiation efficiency]. The CFO-optimized arrays have generally flatter gain curves with moderate gain values compared to the A3 design. While the A3's gain is higher mid-band, the gain bandwidth is narrow, and the gain falls off precipitously with increasing frequency. Both CFO-optimized arrays also exhibit a substantial decrease in gain at the high end of the band, but the drop off occurs well above the highest frequency with acceptable

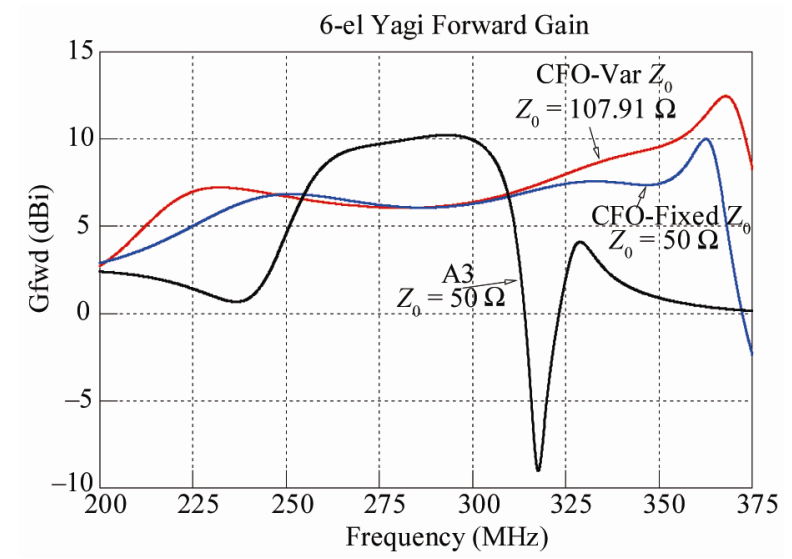

Figure 4. Yagi forward gain.

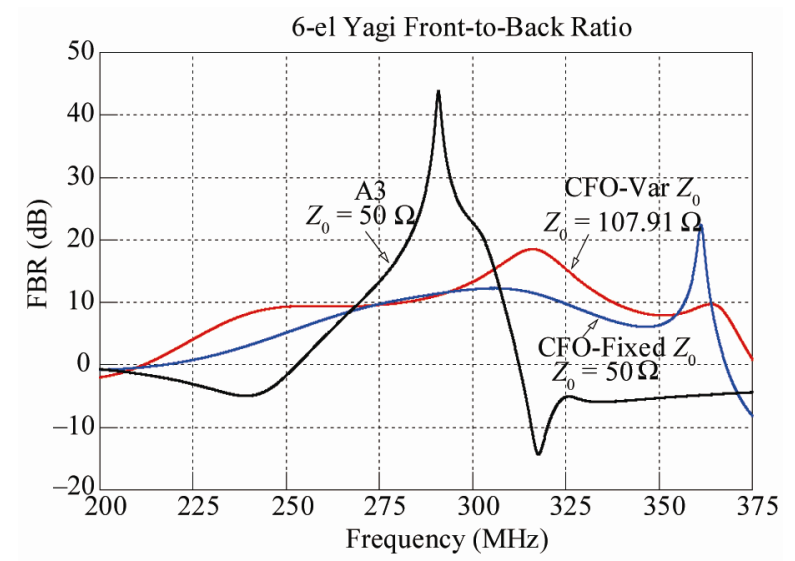

Figure 5. Yagi FBR.
VSWR. The A3 array has a very high mid-band FBR, but only over a fairly narrow range of frequencies. On either side, its FBR decreases sharply and quickly. The CFOoptimized arrays exhibit more moderate FBR values, but over a much greater bandwidth, with the Var $Z_{0}$ design performing better than its Fixed $Z_{0}$ counterpart.

Input resistance and reactance appear in Figures 6 and 7, respectively. The two CFO-optimized arrays exhibit similar behavior for $R_{\text {in }}$ with moderate values across the band, whereas the A3's input resistance drops nearly to zero beyond around $310 \mathrm{MHz}$. For all three antennas, the reactance increases more or less monotonically from about $-110 \Omega$ at $200 \mathrm{MHz}$ to between $+130 \Omega$ and +130 $\Omega$ at $375 \mathrm{MHz}$. Each Yagi exhibits a single resonance across the entire band.

\section{Conclusion}

This paper provides an example of applying Central Force Optimization and Variable $Z_{0}^{(\mathrm{sm})^{*}}$ technology to the design of a wideband Yagi-Uda array. Even though Yagis are generally considered "narrowband" antennas, the CFO-Var $Z_{0}$ approach produces a Yagi design with good gain and FBR over a fractional bandwidth greater

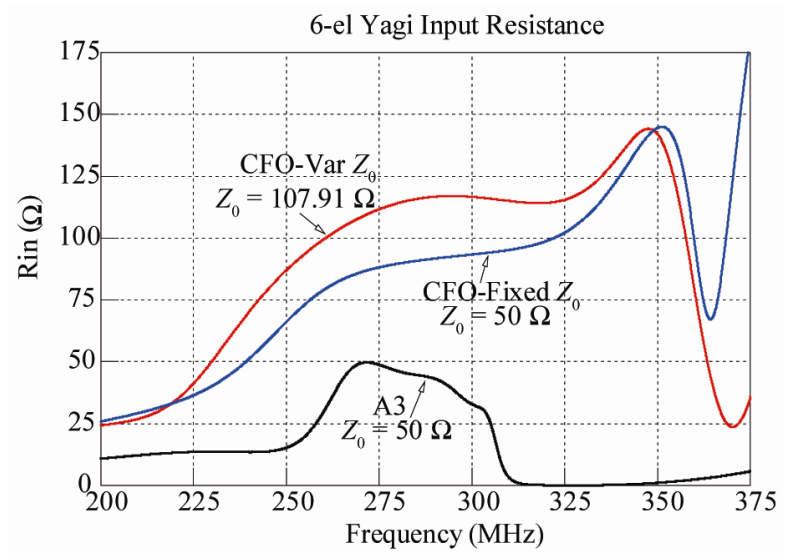

Figure 6. Yagi input resistance.

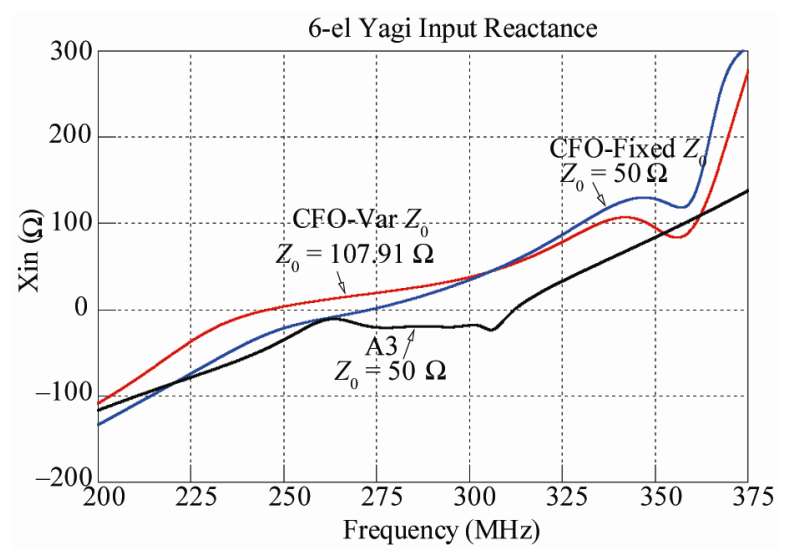

Figure 7. Yagi input reactance. 
than $33 \%$ with $V S W R \leq 2: 1$. Variable $Z_{0}$ is a new approach to antenna design (patent pending) in which the feed system characteristic impedance (or source internal impedance), $Z_{0}$, is treated as a variable quantity whose value is determined by the design or optimization methodology. Variable $Z_{0}$ is a heretofore overlooked and fundamentally different antenna design methodology that departs from the traditional methodology of treating $Z_{0}$ as a fixed design parameter whose value is specified at the outset and never changes. By introducing into the antenna design or decision space an additional degree of freedom, Variable $Z_{0}$ makes it easier to achieve specific performance goals, as illustrated by the Yagi design example. While Variable $Z_{0}$ should be especially useful for improving IBW, it will be useful in achieving any desired antenna performance objectives, even objectives not involving IBW directly.

\section{REFERENCES}

[1] S. Uda, "Wireless Beam of Short Electric Waves," Journal of the Institute of Electrical Engineers (Japan), March 1926, pp. 273-282.

[2] H. Yagi, "Beam Transmission of Ultra Short Waves," $P$ Proceedings of the IEEE, Vol. 85, No. 11, 1928, pp. 1864 -1874 .

[3] C. A. Balanis, "Antenna Theory: Analysis and Design," Harper \& Row, Publishers, New York, 1982.

[4] The ARRL Antenna Book, 20th Edition, American Radio Relay League, Inc., Newington, 2008.

[5] A. C. Lisboa, D. A. G. Vieira, J. A. Vasconcelos, R. R. Saldanha and R. H. C. Takahashi, "Monotonically Improving Yagi-Uda Conflicting Specifications Using the Dominating Cone Line Search Method," IEEE Transactions on Magnetics, Vol. 45, No. 3, 2009, pp. 1494-1497. doi:10.1109/TMAG.2009.2012688

[6] Federal Communications Commission, "Revision of Part 15 of the Commission's Rules Regarding Ultra-Wideband Transmission Systems," First Report and Order, FCC 02-48, Washington DC, 2002.

[7] R. A. Formato, "A Novel Methodology for Antenna Design and Optimization: Variable $Z_{0}$ (ver. 2)," 2011. http://arXiv.org/abs/1107.1437

[8] R. A. Formato, "UWB Array Design Using Variable $Z_{0}$ Technology and Central Force Optimization, ver. 2," 2011. http://arXiv.org/abs/1108.0901

[9] R. A. Formato, "Central Force Optimization: A New Metaheuristic with Applications in Applied Electromagnetics," Progress in Electromagnetics Research, Vol. 77, 2007, pp. 425-491. doi:10.2528/PIER07082403

[10] G. M. Qubati, N. I. Dib, and R. A. Formato, "Antenna Benchmark Performance and Array Synthesis Using Central Force Optimization," Microwaves, Antennas \& Propagation, Vol. 4, No. 5, 2010, pp. 583-592, 2010.

[11] G. Qubati, "Central Force Optimization Method and Its Application to the Design of Antennas," Masters Disser- tation, Jordan University of Science and Technology, Amman, 2010.

[12] G. M. Qubati, and N. I. Dib, "Microstrip Patch Antenna Optimization Using Modified Central Force Optimization," Progress in Electromagnetics Research, Vol. 21, 2010, pp. 281-298.

[13] M. J. Asi and N. I. Dib, "Design of Multilayer Microwave Broadband Absorbers Using Central Force Optimization," Progress in Electromagnetics Research B, Vol. 26, 2010, pp. 101-113. doi:10.2528/PIERB10090103

[14] R. A. Formato, "New Techniques for Increasing Antenna Bandwidth with Impedance Loading," Progress in Electromagnetics Research B, Vol. 29, 2011, pp. 269-288. doi:10.2528/PIERB11021904

[15] R. A. Formato, "Improved CFO Algorithm for Antenna Optimization," Progress in Electromagnetics Research B, Vol. 19, 2010, pp. 405-425. doi:10.2528/PIERB09112309

[16] R. A. Formato, "Parameter-Free Deterministic Global Search with Simplified Central Force Optimization," In: D.-S. Huang, Z. Zhao, V. Bevilacqua and J. C. Figueroa, Eds., Advanced Intelligent Computing Theories and Applications (ICIC2010), Springer-Verlag, Berlin, 2010, pp. 309-318.

[17] R. A. Formato, "Central Force Optimization with Variable Initial Probes and Adaptive Decision Space," Applied Mathematics and Computation, Vol. 217, No. 21, 2011, pp. 8866-8872. doi:10.1016/j.amc.2011.03.151

[18] R. A. Formato, "Central Force Optimization Applied to the PBM Suite of Antenna Benchmarks," 2010. http://arXiv.org/abs/1003.0221.

[19] G. J. Burke, "Numerical Electromagnetics Code-NEC4.2 Method of Moments, Part I: User's Manual," LLNLSM-490875, Lawrence Livermore National Laboratory, Livermore, 2011.

[20] G. J. Burke, "Numerical Electromagnetics Code-NEC-4, Method of Moments, Part I: User's Manual and Part II: Program Description-Theory," UCRL-MA-109338, Lawrence Livermore National Laboratory, Livermore, 1992.

[21] G. J. Burke and A. J. Poggio, "Numerical Electromagnetics Code (NEC) - Method of Moments," Parts I, II and III, UCID-19934, Lawrence Livermore National Laboratory, Livermore, January 1981.

[22] "4nec2" Antenna Modeling Freeware by Arie Voors, 2005. http://home.ict.nl/ arivoors/

[23] Unofficial Numerical Electromagnetic Code (NEC) Archives, http://www.si-list.net/swindex.html

[24] Y. Zehforoosh, C. Ghobadi and J. Nourinia, "Antenna Design for Ultra Wideband Application Using a New Multilayer Structure," PIERS Online, Vol. 2, No. 6, 2006, pp. 544-549. doi:10.2529/PIERS060531145356

[25] R. A. Formato, "Issues in Antenna Optimization-A Monopole Case Study," 2011. http://arXiv.org/abs/1103.5629

[26] J. Kennedy, and R. Eberhart, "Particle Swarm Optimization," Proceeding of IEEE Conference on Neural Networks, Vol. 4, 1995, pp. 1942-1948.

[27] M. Dorigo, V. Maniezzo and A. Colorni, "Positive Feed- 
back as a Search Strategy," Dipartimento di Elettronica, Politecnico di Milano, Italy, Technical Report 91-016, 1991. http://iridia.ulb.ac.be/ mdorigo/pub_x_subj.html

[28] S. He, Q. H. Wu, and J. R. Saunders, "Group Search Optimizer: An Optimization Algorithm Inspired by Animal Searching Behavior," IEEE Trans. Evol. Computation, Vol. 13, No. 5, 2009, pp. 973-990.

[29] R. Storn and K. V. Price, "Differential Evolution: A Simple and Efficient Adaptive Scheme for Global Optimization over Continuous Spaces," Technical Report TR-95012, ICSI (Univ. of California, Berkeley), 1995. http://www.icsi.berkeley.edu/ storn/litera.html

[30] R. Storn and K. V. Price, "Minimizing the Real Functions of the ICEC 1996 Contest by Differential Evolution," Proceedings of 1996 IEEE International Conference on Evolutionary Computation, Nagoya, 20-22 May 1996, pp. 842-844. doi:10.1109/ICEC.1996.542711

[31] A. Chowdhury, A. Ghosh, R. Giri and S. Das, "Optimization of Antenna Configuration with a Fitness-Adaptive Differential Evolution Algorithm," Progress in Electromagnetics Research B, Vol. 26, 2010, pp. 291-319. doi:10.2528/PIERB10080703

[32] E. A. Jones and W. T. Joines, "Design of Yagi-Uda Antennas Using Genetic Algorithms," IEEE Transactions on Antennas and Propagation, Vol. 45, No. 9, 1997, pp. 1386-1392. doi:10.1109/8.623128 